\title{
Finding a Rank-Maximizing Matrix Block
}

\author{
A. J. Goldman and M. Newman \\ Institute for Basic Standards, National Bureau of Standards, Washington, D.C. 20234
}

(May 11, 1966)

\begin{abstract}
An algorithm is developed for the following problem: Given three matrices of respective dimensions $s \times s, s \times t$, and $t \times s$, to find a $t \times t$ matrix such that the $(s+t) \times(s+t)$ matrix formed from the four blocks has maximum rank.
\end{abstract}

Key words: Matrix, block, rank, algorithm.

Let $A, B, C$ be matrices (over any field) of respective dimensions $s \times s, s \times t, t \times s$. We shall show how to find a $t \times t$ matrix $X$ such that

$$
M(X)=\left[\begin{array}{ll}
A & B \\
C & X
\end{array}\right]
$$

has maximum possible rank $\rho(M(X))$, or equivalently has minimum possible deficiency

$$
\delta(M(X))=s+t-\rho(M(X)) .
$$

Let $\beta^{\prime}$ and $\gamma^{\prime}$ be the respective ranks of the matrices

$$
\left[\begin{array}{ll}
A & B
\end{array}\right],\left[\begin{array}{l}
A \\
C
\end{array}\right] \text {. }
$$

It is readily seen that for any choice of $X$,

$$
\delta(M(X)) \geqslant \max \left(s-\beta^{\prime}, s-\gamma^{\prime}\right) .
$$

We will show that $X$ can be chosen so that equality holds in (1). Thus

$$
\min _{X} \delta(M(X))=\max \left(s-\beta^{\prime}, s-\gamma^{\prime}\right) .
$$

For the special case $\beta^{\prime}=\gamma^{\prime}=s$, this was proved by Pearl. ${ }^{1}$

Denote the rank of $A$ by $\alpha$. Find nonsingular $s \times s$ matrices $U$ and $V$ which bring $A$ into Smith normal form, i.e.,

$$
U A V=I_{\alpha} \dot{+} 0_{s-\alpha} .
$$

\footnotetext{
${ }^{1}$ Martin Pearl, On Normal EPr Matrices, Mich. Math. J. 8, 33-37 (1961), (Theorem 2).
}

$M(X)$ will have the same rank and deficiency as

$$
M_{1}(X)=\left[\begin{array}{ll}
U & 0 \\
0 & I
\end{array}\right] M(X)\left[\begin{array}{ll}
V & 0 \\
0 & I
\end{array}\right]=\left[\begin{array}{ll}
U A V & U B \\
C V & X
\end{array}\right]
$$

which we repartition as

$$
M_{1}(X)=\left[\begin{array}{lll}
I_{\alpha} & 0 & B_{1} \\
0 & 0_{s-\alpha} & B_{2} \\
C_{1} & C_{2} & X
\end{array}\right]
$$

where

$$
\left[C_{1} C_{2}\right]=C V,\left[\begin{array}{l}
B_{1} \\
B_{2}
\end{array}\right]=U B
$$

$M_{1}(X)$, in turn, has the same rank and deficiency as

$$
M_{2}(X)=\left[\begin{array}{lll}
I & 0 & 0 \\
0 & I & 0 \\
-C_{1} & 0 & I
\end{array}\right] M_{1}(X)\left[\begin{array}{lll}
I & 0 & -B_{1} \\
0 & I & 0 \\
0 & 0 & I
\end{array}\right]
$$

$$
=\left[\begin{array}{lll}
I & 0 & 0 \\
0 & 0 & B_{2} \\
0 & C_{2} & X-C_{1} B_{1}
\end{array}\right] .
$$

Let $\beta$ and $\gamma$ be the respective ranks of $B_{2}$ and $C_{2}$. Since $\left[\begin{array}{ll}A & B\end{array}\right]$ has the same rank as

$$
U\left[\begin{array}{ll}
A & B
\end{array}\right]\left(V+I_{t}\right)\left[\begin{array}{lll}
I & 0 & -B_{1} \\
0 & I & 0 \\
0 & 0 & I
\end{array}\right]=\left[\begin{array}{lll}
I_{\alpha} & 0 & 0 \\
0 & 0 & B_{2}
\end{array}\right]
$$

we see that $\beta^{\prime}=\alpha+\beta$, and similarly $\gamma^{\prime}=\alpha+\gamma$.

Find a nonsingular $(s-\alpha) \times(s-\alpha)$ matrix $U_{2}$ and a nonsingular $t \times t$ matrix $V_{2}$ which together bring $B_{2}$ into Smith normal form, i.e.,

$$
U_{2} B_{2} V_{2}=\left[\begin{array}{ll}
I_{\beta} & 0 \\
0 & 0
\end{array}\right] .
$$


Then $M_{2}(X)$ has the same rank and deficiency as

$$
\begin{aligned}
M_{3}(X) & =\left[\begin{array}{lll}
I & 0 & 0 \\
0 & U_{2} & 0 \\
0 & 0 & I
\end{array}\right] M_{2}(X)\left[\begin{array}{lll}
I & 0 & 0 \\
0 & I & 0 \\
0 & 0 & V_{2}
\end{array}\right] \\
& =\left[\begin{array}{lll}
I & 0 & 0 \\
0 & 0 & U_{2} B_{2} V_{2} \\
0 & C_{2} & \left(X-C_{1} B_{1}\right) V_{2}
\end{array}\right] .
\end{aligned}
$$

Next find a nonsingular $t \times t$ matrix $U_{3}$ and a nonsingular $(s-\alpha) \times(s-\alpha)$ matrix $V_{3}$ which together bring $C_{2}$ into Smith normal form, i.e.,

$$
U_{3} C_{2} V_{3}=\left[\begin{array}{cc}
I \gamma & 0 \\
0 & 0
\end{array}\right]
$$

Then $M_{3}(X)$ has the same rank and deficiency as

$$
\begin{gathered}
M_{4}(X)=\left[\begin{array}{lll}
I & 0 & 0 \\
0 & I & 0 \\
0 & 0 & U_{3}
\end{array}\right] M_{3}(X)\left[\begin{array}{lll}
I & 0 & 0 \\
0 & V_{3} & 0 \\
0 & 0 & I
\end{array}\right] \\
=\left[\begin{array}{lll}
I & 0 & 0 \\
0 & 0 & U_{2} B_{2} V_{2} \\
0 & U_{3} C_{2} V_{3} & U_{3}\left(X-C_{1} B_{1}\right) V_{2}
\end{array}\right],
\end{gathered}
$$

which we repartition as

$$
M_{4}(X)=\left[\begin{array}{lllll}
I_{\alpha} & 0 & 0 & 0 & 0 \\
0 & 0 & 0 & I_{\beta} & 0 \\
0 & 0 & 0 & 0 & 0 \\
0 & I_{\gamma} & 0 & Y_{1} & Y_{2} \\
0 & 0 & 0 & Y_{3} & Y_{4}
\end{array}\right],
$$

where $\quad U_{3}\left(X-C_{1} B_{1}\right) V_{2}=\left[\begin{array}{ll}Y_{1} & Y_{2} \\ Y_{3} & Y_{4}\end{array}\right]$.

At this point $\beta$ and $\gamma$ are known. Assume without loss of generality that $\beta \leqslant \gamma$. Choose $Y_{1}=0, Y_{2}=0$,
$Y_{3}=0$, and choose the $(t-\gamma) \times(t-\beta)$ matrix $Y_{4}=\left[I_{t-\gamma} 0\right]$. On the one hand, this choice yields

$$
\begin{aligned}
\rho(M(X))=\rho\left(M_{4}(X)\right) & =\alpha+\gamma+\beta+(t-\gamma) \\
= & \alpha+t+\min (\beta, \gamma)=t+\min \left(\beta^{\prime}, \gamma^{\prime},\right),
\end{aligned}
$$

so that equality holds in (1). On the other hand the choice is achieved by setting

$$
X=C_{1} B_{1}+U_{3}^{-1}\left[\begin{array}{lll}
0 & 0 & 0 \\
0 & I_{t-\gamma} & 0
\end{array}\right] V_{2}^{-1} .
$$

If $\gamma<\beta$, then (4) can be replaced by

$$
X=C_{1} B_{1}+U_{3}^{-1}\left[\begin{array}{cc}
0 & 0 \\
0 & 0 \\
0 & I_{t-\beta}
\end{array}\right] V_{2}^{-1} ;
$$

i.e., $Y_{1}=0, Y_{2}=0, Y_{3}=0, Y_{4}^{T}=\left[\begin{array}{ll}0 & I_{t-\beta}\end{array}\right]$.

The main computational labor involved comes (a) in bringing $A$ into Smith normal form, (b) in determining $V_{2}^{-1}$ and $\beta$, and (c) in determining $U_{3}^{-1}$ and $\gamma$. Step (a) can be carried out by performing elementary row operations to "sweep out" $A$ to upper triangular form (the product of these operators' matrices is $U$ ), and then performing elementary column operations (the product of whose matrices is $V$ ) to "sweep out" the resulting matrix into diagonal form $D_{\alpha}+0_{s-\alpha}$; it is not necessary to normalize $D_{\alpha}$ to $I_{\alpha}$. As for step (b), we can take $V_{2}$ as the product of the matrices of elementary column operations used to sweep out $B_{2}$ into lower triangular form; each elementary matrix is trivially invertible so that $V_{2}^{-1}$ can be built up during the sweepout process, and $\beta$ can be read off at the end of the process. Similarly for step (c).

(Paper 70B3-183) 